\title{
Strates
}

STRATES Matériaux pour la recherche en sciences sociales

Hors-série | 2002

Parcours dans la recherche urbaine, Michel Rochefort, un géographe engagé

\section{Des villes et des campagnes à l'ombre de Paris}

\section{Martine Berger et Thierry Saint-Gérand}

\section{(2) OpenEdition \\ Journals}

Édition électronique

URL : http://journals.openedition.org/strates/578

DOI : $10.4000 /$ strates. 578

ISSN : 1777-5442

Éditeur

Laboratoire Ladyss

Édition imprimée

Date de publication : 1 janvier 2002

ISSN : 0768-8067

Référence électronique

Martine Berger et Thierry Saint-Gérand, "Des villes et des campagnes à l'ombre de Paris », Strates [En ligne], Hors-série | 2002, mis en ligne le 19 mai 2005, consulté le 26 octobre 2020. URL : http://

journals.openedition.org/strates/578; DOI : https://doi.org/10.4000/strates.578

Ce document a été généré automatiquement le 26 octobre 2020

Tous droits réservés 


\title{
Des villes et des campagnes à l'ombre de Paris
}

\author{
Martine Berger et Thierry Saint-Gérand
}

1 Pour nous qui avons suivi les cours de Michel Rochefort à l'Institut de géographie de Paris, et découvert à la lecture de sa thèse l'existence des réseaux urbains, ce fut la révélation qu'une autre approche des villes et des régions était possible. Tranchant avec la manière très monographique et descriptive des études régionales, Michel Rochefort nous proposait de décortiquer et de reconstruire le système des relations entre villes et campagnes. À l'assemblage empiriste succédaient la rigueur et la cohérence d'une méthode d'analyse des interrelations entre les territoires transposable en différents points du globe. Villes et régions cessaient d'être uniques et insulaires, une géographie générale des espaces régionaux et des organismes urbains devenait possible. Un vent nouveau soufflait, une démarche nous était proposée pour sortir de l'accumulation des monographies. Et, sans renier les acquis antérieurs - l'approche fonctionnaliste des villes, la combinaison des échelles, ou la reconnaissance du poids de l'action des hommes -, elle intégrait les apports de l'économie spatiale, et rendait compte de la rapidité des transformations du territoire français dans l'immédiat aprèsguerre qui voit la consécration de l'importance des activités tertiaires.

2 Osons avouer que la «méthode expérimentale» de Michel Rochefort, son souci de tester systématiquement les indicateurs les plus fiables et les plus robustes, les plus susceptibles d'un emploi généralisé au-delà de la diversité des territoires et de leurs situations ${ }^{1}$, nous ont séduit plus durablement que les approches plus théoriques que d'autres ont développées dans les années soixante-dix. Dans leur objectif de mettre en équation les régularités, ces dernières ont trop souvent réduit l'espace à de l'étendue, même si elles l'affectent de masses de population ou de flux d'échanges. Car l'analyse régionale que Michel Rochefort nous incitait à pratiquer n'oubliait pas les acteurs, leurs différents statuts et professions, leur inscription dans l'espace et leurs aires de déplacements. Nous apprenions à lire dans le tissu urbain la hiérarchie des fonctions et les structures sociales : nous étions confrontés à leur complexité spatiale. Les villes d'Alsace et d'ailleurs prenaient une densité, une couleur, qui ne tenait pas seulement à 
la richesse de leur ancrage régional, mais aussi à l'inscription dans leur paysage de la mémoire des fonctions assumées autrefois. Ces pôles, ces métropoles symboles de modernité, avaient encore une âme, celle que leur avaient léguée des générations d'habitants, des tisserands aux notaires, des médecins aux chefs d'agences bancaires et aux capitaines d'industrie.

Bien loin d'une vision passéiste ou d'un recours à l'explication génétique pour éluder la recherche des mécanismes économiques, sociaux ou politiques qui contribuent à la production et à l'évolution des territoires, la restitution d'états antérieurs des systèmes urbains de l'Alsace ou de l'État de São Paulo met en évidence les facteurs et mécanismes de passage d'un état à un autre des relations entre villes et campagnes. La description de situations historiques est utilisée comme un substitut de la méthode expérimentale, un moyen de pratiquer la comparaison. La succession des expériences spatiales des sociétés permet de révéler les principes, sinon les lois, d'évolution des régions. Entre la description de l'alignement urbain sous-vosgien et celle du semis des places de marché dans le sud du Dakota ${ }^{2}$, les postulats et les méthodes ne sont pas si éloignés qu'ont parfois voulu le faire croire, dans leur zèle refondateur, certains tenants de la géographie théorique et quantitative. L'espace porte partout la marque de structures d'âges différents, dont la persistance perturbe la régularité des modèles théoriques. L'analyse de ces trames héritées permet de valider les lois générales d'organisation des systèmes de places centrales. L'Alsace des années cinquante, le Brésil du front pionnier pauliste ou la France des années soixante offrent l'opportunité de réfléchir aux principes généraux d'organisation des relations villes campagnes et aux rapports entre pôles de niveaux hiérarchiques différents.

4 Cette conviction qu'il est possible de développer des méthodes de recherche généralisables, au delà de la diversité des terrains d'enquête, Michel Rochefort l'a défendue aussi en créant et en animant le Laboratoire de géographie humaine, où se sont côtoyés, dès le début des années soixante-dix, "urbains » et "ruralistes ", «hexagonaux» et "tiers-mondistes». Après plus d'un quart de siècle de "vie commune ", il nous semble que le pari d'un pluralisme des terrains valait largement la peine d'être tenté, et que l'échange des expériences dans cette aventure commune a permis à beaucoup d'entre nous d'assumer les changements de cap qu'implique tout itinéraire de recherche. Le refus d'étiquettes réductrices et la confrontation de problématiques et de méthodes testées sur des types d'espaces différents ont constitué pour nous l'apport essentiel de ce laboratoire. De l'équipe « Rurale » à « l'observatoire du changement métropolitain ", nous avons pu y développer une approche des espaces périurbains parisiens s'intégrant dans un projet global d'exploration des rapports entre villes et campagnes qui associe l'analyse des systèmes productifs à celle des systèmes sociaux.

5 Nous ne présenterons pas ici les résultats de travaux sur la grande Région parisienne menés en parallèle au sein du laboratoire avec ceux de F. Damette et P. Beckouche (sur les systèmes productifs urbains) ou de J.P. Charvet et M. Poulot (sur les systèmes d'exploitation agricoles et leur encadrement). Notre propos est de souligner combien notre analyse des espaces périurbains est redevable aux perspectives ouvertes par M. Rochefort tant dans sa thèse sur l'Alsace que dans sa lecture de l'évolution de l'armature urbaine française ${ }^{3}$.

6 Situé dans l'ombre de Paris, l'espace du proche Bassin parisien se caractérise par la combinaison d'une organisation en couronnes, effet de la distance à la capitale, et par 
un pavage en zones d'influence de villes de 2e ou 3e rang qui ne peuvent se développer qu'hors de la portée directe d'une très grande métropole. Les fonctions de ces différents niveaux de villes, ainsi que les perturbations apportées à l'organisation des réseaux par la proximité de Paris ont été décrites par de nombreux auteurs ${ }^{4}$. Le(s) système(s) productif(s) et le(s) système(s) urbain(s) de ces villes sous forte influence parisienne sont désormais bien connus. Mais comment s'organisent les espaces interstitiels - ruraux et périurbains - dans l'arrière-pays de la capitale? Le dispositif des navettes domicile-travail, le niveau de qualification des emplois, ainsi que la structure sociale des populations résidentes permettent d'apporter quelques réponses à ces questions.

7 Si certaines de ces villes ont des fonctions atrophiées et incomplètes et connaissent une dégradation du rapport entre le nombre d'actifs résidents et l'offre d'emplois, ceci n'exclut pas l'élargissement de leurs couronnes périurbaines et le renforcement de leur rôle polarisateur en termes de navettes quotidiennes, comme le montrent les cartes figurant les navettes de 5 à $14 \mathrm{~km}$. En dépit des difficultés qu'elles rencontrent du fait de la crise du modèle de partage du travail qui avait présidé aux décentralisations industrielles, la plupart des villes du Bassin parisien ont renforcé leur attraction sur l'espace rural environnant et élargi leur bassin d'emploi. Dans les huit départements qui bordent l'Ile-de-France, deux ruraux sur trois travaillaient à la campagne en 1975 ; il n'en reste qu'un sur deux en 1990. Les campagnes du Bassin parisien proche ont gagné en 15 ans 120000 actifs (plus des $2 / 3$ de la croissance régionale) et perdu 16000 emplois alors que les villes gagnaient trois fois plus d'emplois que d'actifs. La raréfaction de l'emploi rural et l'installation périurbaine de nombreuses familles ont fortement contribué au renforcement des relations quotidiennes entre villes et campagnes.

Dans le même temps, l'attraction de Paris et des pôles d'emploi franciliens s'étend et s'accroît: ils prélèvent de plus en plus d'actifs, et de plus en plus loin, dans les départements limitrophes, comme le montrent les cartes des navettes de $15 \mathrm{~km}$ et plus. Cette attraction s'exerce sur les plus qualifiés des actifs régionaux, sensibles au niveau des salaires franciliens et à l'ampleur du marché d'emploi de la capitale. Mais elle concerne aussi des catégories sociales modestes, que les coûts d'accession à la propriété en Île-de-France ont conduit à faire le choix de longues navettes quotidiennes. Tant du point de vue des emplois qui s'y localisent que des actifs qui y résident, le Bassin parisien proche fait donc figure d'arrière-cour de la capitale. $\mathrm{Si}$, à l'échelle de l'ensemble du territoire national, il se situe dans l'auréole de richesse émanant de la capitale ${ }^{5}$, plusieurs décennies de déconcentration des fonctions d'exécution et de pompage des cadres, et, plus récemment, le redéploiement résidentiel d'ouvriers et d'employés franciliens ont contribué à la mise en place d'un dispositif spatial en gradient opposant fortement centre et périphérie. Malgré la persistance d'une forte dissymétrie des dynamiques démographiques et économiques entre l'est et l'ouest de la région, l'organisation en secteurs cardinaux socialement différenciés, caractéristique du centre de la région parisienne, s'estompe au delà de $60 \mathrm{~km}$ de la capitale. La distance à Paris devient le facteur essentiel de la division sociale de l'espace, en ville comme à la campagne.

9 Est-ce à dire que la concurrence parisienne, surtout lorsqu'elle s'exerce sur des espaces peu densément peuplés, bien désenclavés, ou dont les résidents disposent d'un certain niveau de revenus, rend problématique l'existence d'une organisation régionale fondée 
sur la densité des relations entre les villes et les campagnes qui les entourent? Sans prétendre répondre ici à cette difficile question, nous voudrions souligner ce qui nous semble caractériser l'évolution du système urbain parisien. Les grandes exploitations agricoles qui vivent au rythme des marchés mondiaux se sont affranchies des tutelles foncières et commerciales des villes proches, d'autant que la solvabilité de leur clientèle ne pouvait compenser le handicap que constituait, pour l'implantation de commerces et de services dans ces villes, leur environnement de faibles densités. Mais si l'agriculteur ne se rend plus dans la ville la plus proche pour négocier ses récoltes et ses crédits ou acheter ses machines, son épouse et ses enfants y vont chaque jour pour leur travail, leurs études, ou leurs achats. La quotidienneté de cette expérience, la banalité des services offerts ont sans doute diminué le prestige des villes. Mais la concentration croissante des emplois, des commerces et des services qui s'y opère n'at-elle pas renforcé leur pouvoir de commandement?

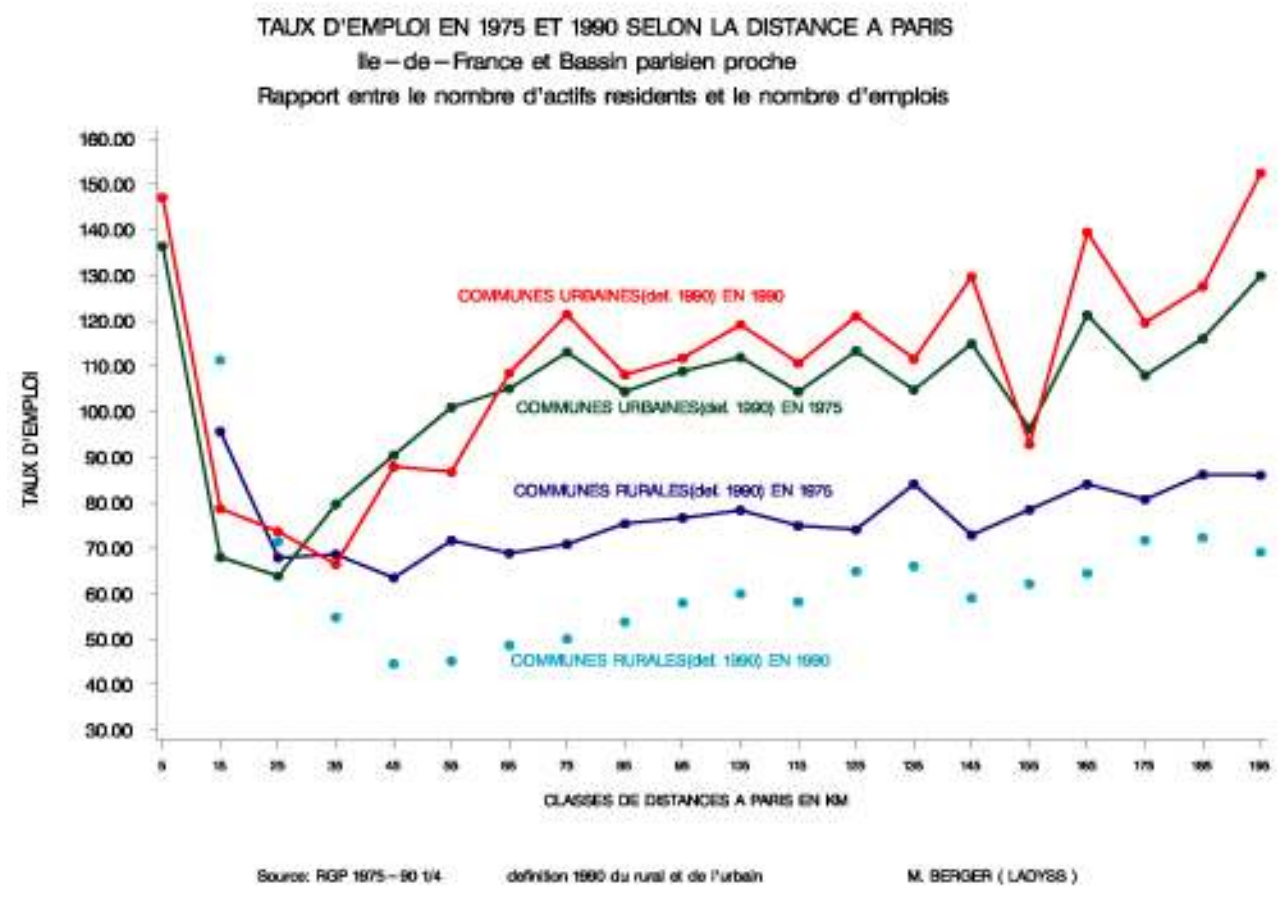



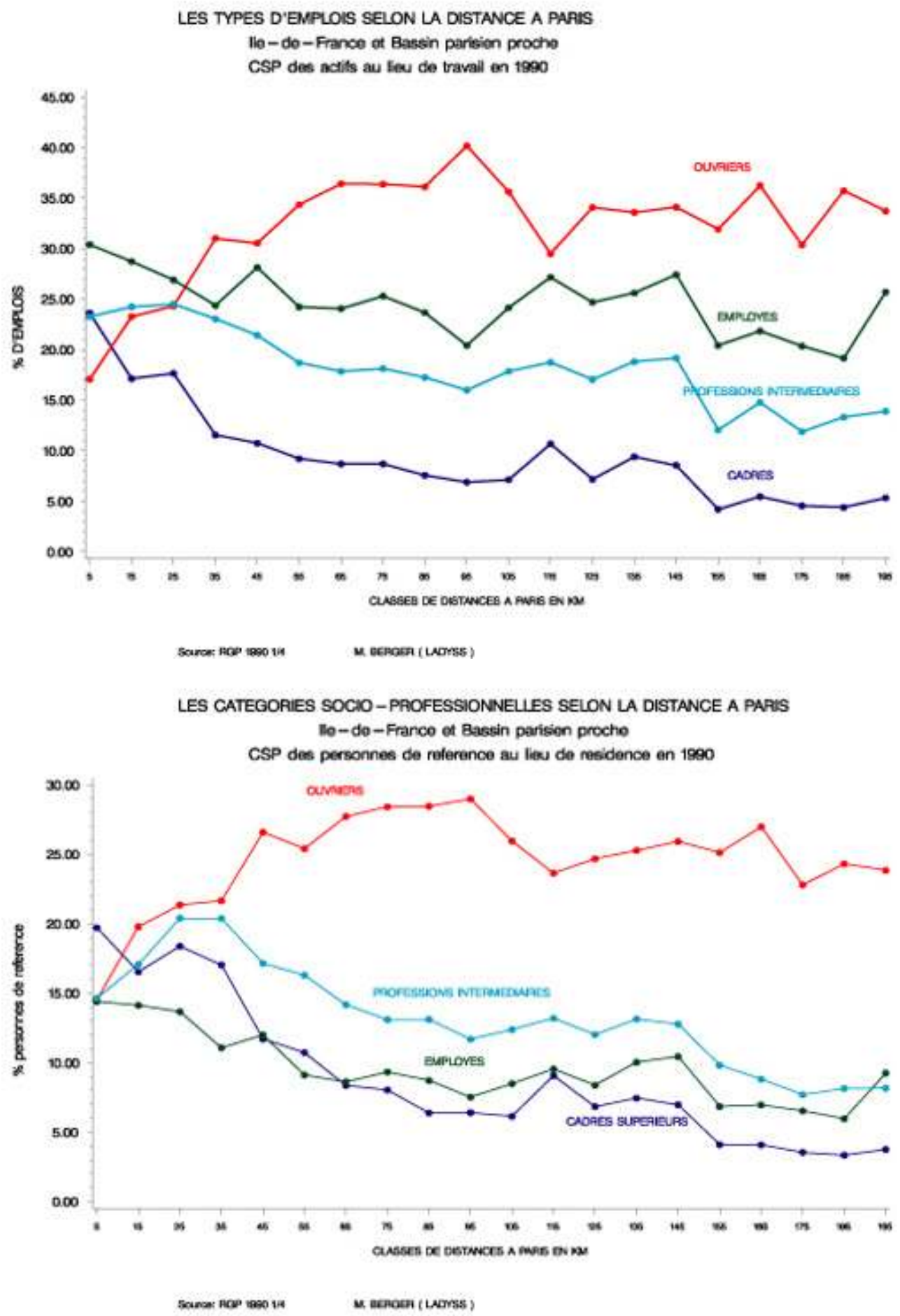

Emplois et groupes sociaux gardent leurs distances

Certes la contraction de l'espace-temps - qui résulte de l'amélioration des systèmes de communication particulièrement sensible à proximité du nœud parisien - a sans doute accentué le déclassement des niveaux moyens de la hiérarchie urbaine et les chevauchements entre leurs aires d'influence. Mais la généralisation de l'automobile, la bi-motorisation de plus en plus fréquente des ménages, n'ont pas effacé le rôle de la 
distance à la ville dans l'organisation des espaces, qu'il s'agisse des pôles urbains proches ou de la capitale. Les emboîtements ont sans doute laissé la place à des recouvrements d'aires d'influence, les hiérarchies entre les villes à des concurrences. Mais il existe bien toujours différentes échelles de polarisation de l'espace par les villes, différentes portées de leur action, comme en témoignent le dispositif des navettes quotidiennes et la division sociale de l'espace, qu'il s'agisse de la qualification des emplois ou des groupes sociaux des résidents. À l'ombre de Paris, villes et campagnes du Bassin parisien n'échappent pas pour autant aux lois qui règlent l'organisation de l'espace dans les pays industriels.

\section{NOTES}

1.M. Rochefort (1957), « Méthodes d'étude des réseaux urbains. Intérêt de l'analyse du secteur tertiaire de la population active ». Annales de Géographie.

2.B. J. L. Berry (1967), Geography of market centers and retail distribution. Prentice Hall, Englewood Cliffs, N. J. (traduction française : Paris, A.Colin, 1971).

3.Cf. en particulier: M. Rochefort (1967), « Les notions de réseau urbain et d'armature urbaine », Geographia polonica, 12, et M. Rochefort (1993), « Des métropoles d'équilibre aux métropoles d'aujourd'hui » Métropoles en déséquilibre ? Paris, Economica.

4.Cf. par exemple : P. Oudart (1982), Les grandes villes de la couronne urbaine de Paris, de la Picardie à la Champagne; M. Michel (1984), Développement des villes moyennes: Chartres, Dreux, Evreux. Paris, Publications de la Sorbonne, 2 t.

5.Cf. par exemple les cartes figurant le revenu moyen par foyer fiscal, in Données Urbaines, 1996, D. Pumain et F. Godard (ed.), p 149.

\section{AUTEURS}

\section{MARTINE BERGER}

Maître de conférences, université de Paris I, Ladyss 